\title{
Optimization of Shouwu Fang in SH-SY5Y cell lines by uniform design
}

\author{
Yang Liu, Yue Jiao, Nan Ya Sun, Lan Ying Nie, Yan Yan Ma, Mao Yu Jiang, Liang Xiao Zhao, \\ Tao Li, Yu Mei Zhang, Qiao Dan Wang \\ Experimental Research Center, China Academy of Chinese Medical Sciences, China
}

Shouwu Fang (SWF) is clinically effective Chinese compound used with L-DOPA for Parkinson's disease. Studies have shown its mechanism may relate to its regulation on cerebral amino acids. However, Heshouwu (Fleeceflower Root), as the main medicinal in the compound, has been debated for its safety recent years. The compound would be further optimized to ensure its safety and efficacy. To optimize ingredients in SWF (Fleeceflower Root, Hairy Deer-horn, Szechwan Lovage Rhizome, Magnolia officinalis, Tall Gastrodis Tuber, and Gambir Plant), uniform design was adopted to design doses of Hairy Deer-horn, Szechwan Lovage Rhizome, Magnolia officinalis, Tall Gastrodis Tuber, and Gambir Plant. According to the design, there are total 12 compounds with no Fleeceflower Root with different doses. The compounds were made into powders by freeze drying from decoction of raw medicinals. Cell death was quantified in SHSY5Y cell lines by measurement of 3-(4,5-dimethylthiazol-2-yl)-2,5-diphenyl-tetrazolium bromide (MTT) at 24-hour exposing to different compounds to verify effective and harmless concentrations. Viability of MPP+ (1-methyl-4-phenylpyridinium)-treated SHSY5Y cells were was analyzed using cell counting kit-8 assay to evaluate protective efficacy of 13 compounds at the same final concentration. Annexin V-FITC/PI flow cytometry was used to detect the rate of MPP+ induced apoptosis and apoptosis cycle after 24-hour exposing to the most effective compound in SHSY5Y cell lines. Some compounds with no Fleeceflower Root also promoted cell multiplication and prevented MPP+- induced apoptosis. Further study is necessary to evaluate these compounds in rat model of Parkinson's disease. 